\title{
Fast laser surface texturing of spherical samples to improve the frictional performance of elasto-hydrodynamic lubricated contacts
}

\author{
G. BOIDI ${ }^{1,2,3, *}$, P.G. GRÜTZMACHER ${ }^{4,5}$, A.KADIRIC ${ }^{1}$, F.J. PROFITO ${ }^{3}$, I.F. MACHADO ${ }^{3}$, C. GACHOT $^{4}$, \\ D. DINI ${ }^{1}$
}

${ }^{1}$ Department of Mechanical Engineering, Imperial College London, South Kensington Campus, London SW7 2AZ, UK

${ }^{2}$ AC2T research GmbH, Wiener Neustadt 2700, Austria

${ }^{3}$ Polytechnic School, University of São Paulo-USP, São Paulo 05508-030, Brazil

${ }^{4}$ Department of Engineering Design and Product Development, TU Wien, Wien 1060, Austria

${ }^{5}$ Department of Material Science and Engineering, Saarland University, Saarbrücken 66123, Germany

Received: 17 June 2020 / Revised: 10 September 2020 / Accepted: 14 October 2020

(C) The author(s) 2020.

\begin{abstract}
Textured surfaces offer the potential to promote friction and wear reduction by increasing the hydrodynamic pressure, fluid uptake, or acting as oil or debris reservoirs. However, texturing techniques often require additional manufacturing steps and costs, thus frequently being not economically feasible for real engineering applications. This experimental study aims at applying a fast laser texturing technique on curved surfaces for obtaining superior tribological performances. A femtosecond pulsed laser (Ti:Sapphire) and direct laser interference patterning (with a solid-state Nd:YAG laser) were used for manufacturing dimple and groove patterns on curved steel surfaces (ball samples). Tribological tests were carried out under elasto-hydrodynamic lubricated contact conditions varying slide-roll ratio using a ball-on-disk configuration. Furthermore, a specific interferometry technique for rough surfaces was used to measure the film thickness of smooth and textured surfaces. Smooth steel samples were used to obtain data for the reference surface. The results showed that dimples promoted friction reduction (up to 20\%) compared to the reference smooth specimens, whereas grooves generally caused less beneficial or detrimental effects. In addition, dimples promoted the formation of full film lubrication conditions at lower speeds. This study demonstrates how fast texturing techniques could potentially be used for improving the tribological performance of bearings as well as other mechanical components utilised in several engineering applications.
\end{abstract}

Keywords: surface laser texturing; elasto-hydrodynamic lubrication; bearings; friction; film thickness

\section{Introduction}

Surface modifications for improving tribological performances of machine elements have been used since the 1950s [1]. The introduction of cross-patterns in cylinder liners could improve lubrication and prevent seizure, especially in diesel engines [2].
Additionally, surface texturing was used for preventing adhesion in manufacturing operations [3], and more recently, it was extensively applied for improving lubricant load-carrying capacity [4-6]. The tribological effects of surface texturing are still a common research topic scrutinised by many scientists, and the number of papers related to this

* Corresponding author: G. BOIDI, E-mail: Guido.Boidi@ac2t.at 
topic has recently risen significantly [7]. Some authors even ventured to apply machine learning tools for predicting the coefficient of friction on textured surfaces [8].

Generally, surface textures can be applied by material removal, addition, or plastic deformation [9]. Most common techniques for tribological applications are based on removing material since surface cavities could behave as debris traps for wear reduction [10-12] and oil reservoirs for improving lubrication $[13,14]$. Divergent and convergent regions of surface features could increment lubricant loadcarrying capacity due to the inlet suction $[5,15]$ and micro-hydrodynamic bearing mechanisms [16-19]. Furthermore, cavities offer the advantage of reducing the lubricant shear-strain rate and the contact pressures over the cavities, thus improving the wear and friction behaviour in comparison with protruding surface features [20]. However, shallow texture configurations could wear out during operation and consequently lose their beneficial tribological effects. Additional manufacturing steps and costs also preclude the application of surface textures in some real applications.

Laser texturing is the most successful texturing technique to create surface cavities [21]. It is commonly applied nowadays for designing complex and precise geometries through material ablation. The use of a high-frequency laser with pulse durations in the femtosecond regime permitted to reduce bulges around surface feature edges, improving lubrication conditions. However, precisely tailored features generally require additional processes and costs, restraining the large-scale manufacturing of textured components. For these reasons, the use of fast texturing techniques could significantly decrease the manufacturing costs, favouring the use of texturing techniques in real machine elements. One technique, which can meet these requirements, is Direct Laser Interference Patterning (DLIP). In DLIP, the standing wave pattern created by overlapping two or more laser beams is used to alter the surface topography of different materials periodically. In contrast to conventional laser writing systems where every surface feature has to be created individually, DLIP allows generating a pattern with one single laser pulse. This leads to a significant increase in production speed, allowing to process of materials with speed as high as $1 \mathrm{~m}^{2} / \mathrm{min}$ [22].

Additionally, recent developments in the DLIP technique allow the fabrication of surface textures with feature sizes in the nanometre scale $(<100 \mathrm{~nm})$ [22]. A drawback of DLIP is the shape of the patterns since they are generally defined in a long-range order [22]. Even though by changing the number of interfering beams and their angle to each other, a wide variety of different shapes and periodicities can be created, the degree of freedom is limited compared to laser writing processes. Additionally, the laser textures might show deviations from a perfect geometry, especially for tiny texture features due to the occurrence of melt and a non-perfect overlapping of individual laser spots [23].

Texture performance depends on a wide range of parameters related to the arrangement and geometrical characteristics of features, as well as to operational and environmental conditions. Therefore, it is challenging to design a surface texture configuration that optimizes tribological performance under different working conditions [24]. For these reasons, several researchers strived to create guidelines for designing new texture configurations $[7,9,25,26]$. One major factor influencing the optimum texture design is the acting lubrication regime. For elasto-hydrodynamic lubrication (EHL), there have been several studies focusing on surface textures. For instance, the effect of transversal ridges and the lubricant mechanism involved under sliding/rolling conditions were studied in Refs. [27, 28]. Ehret et al. [29] also scrutinised the effect of surface waviness orientation on film formation. The authors demonstrated that transverse grooves generally improve lubrication, whereas more longitudinal oriented waviness decreases the film thickness. Marian et al. [30] stressed the importance of the relative position between an individual dimple and the direction of the rubbing surface, considering both experimental and numerical approaches. Despite design guidelines for surface texturing and the increased popularity of laser texturing, there are still some aspects regarding their tribological behaviour that have not been deeply understood, such as those related 
to EHL conditions [31-34].

Laser texturing is easily applied on flat and smooth surfaces $[10,11,35]$ since it is possible to control the texture depth and their spatial distribution precisely. However, several mechanical components in real applications present curved geometries, such as journal and rolling element bearings, gears, and cam-tappets, among others. In this study, the possibility of applying surface texturing to curved surfaces using femtosecond pulse laser and DLIP methods was explored to evaluate the feasibility of using these techniques in real engineering components. The scope of this study was to assess the quality of the textured surfaces generated with different techniques and their effect on the overall performance of textured components. In terms of ball rolling elements, it is known that the presence of surface textures may significantly decrease their fatigue life, especially in highly loaded configurations, since textured microgeometries can potentially act as stress concentrators [26]. However, this paper focuses on the application of surface texturing on curved geometries, in components subjected to relatively low EHL contact conditions, and its influence on the frictional and film thickness aspects rather than fatigue life.

This investigation exploits the possibility of using a fast texturing technique (i.e., DLIP) on steel balls, used here to simulate a prototypical contact involving curved surfaces and standard materials encountered in engineering applications (i.e., ball bearings), to obtain superior tribological performance. The tribological effects of surface texture were scrutinised under lubricated point contact varying entrainment speed and sliding-rolling ratio to study different EHL regimes and operational conditions. Furthermore, an interferometry technique designed to measure film thickness in rough surfaces was used for evaluating film thickness on textured curved surfaces.

\section{Materials and methods}

\subsection{Sample materials}

Chromium hardened steel (AISI 52100) ball and disk-shaped specimens were used for the tribological tests. Drilled balls were $19.05 \mathrm{~mm}$ in diameter and disks were $6 \mathrm{~mm}$ in thickness and $46 \mathrm{~mm}$ in diameter. The samples had a smooth surface with a roughness of $S a=6 \mathrm{~nm}$ and $S q=20 \mathrm{~nm}$. Both disk and ball were purchased from the tribometer manufacturer (PCS Instruments); thus, they presented standard controlled dimensions and surface conditions (polished). The surface texture was applied on steel ball samples in the configuration of dimples and longitudinal and perpendicular grooves. Details of the texturing technique used can be found in the next section. An untextured ball and disk pair was utilised as a reference, and it is defined here as Normal Production (NP).

Synthetic base oil (PAO 6) was used for all tribological tests. Main lubricant properties are summarised in Table 1.

\subsection{Texturing manufacturing technique}

Dimple and groove patterns were textured on ball samples. Thereby, the grooves were arranged either longitudinally or perpendicularly to the sliding direction, resulting in four different texture configurations, see Fig. 1. The acronyms FL and FP stand for Full Longitudinal and Full Perpendicular, highlighting the feature orientation related to the relative motion of the ball and the disk. The surface textures were designed based on the most promising solutions suggested in Refs. [7, 9, 24-26], considering the configurations and dimensions that could potentially improve tribological performance in EHL contacts. However, when using fast texturing techniques, a compromise between optimal and suitable configuration for both reducing manufacturing time and obtaining superior tribological performance was assumed.

For the tribological tests, different surface textures were designed to investigate the effect of the texture geometry and manufacturing process. Therefore, two laser systems were used: an Nd : YAG laser

Table 1 Density and kinematic viscosity of lubricant PAO 6 at 40 and $100{ }^{\circ} \mathrm{C}$.

\begin{tabular}{cccc}
\hline Lubricant & $\begin{array}{c}\text { Temperature } \\
\left({ }^{\circ} \mathrm{C}\right)\end{array}$ & $\begin{array}{c}\text { Kinematic } \\
\text { viscosity }\left(\mathrm{mm}^{2} / \mathrm{s}\right)\end{array}$ & $\begin{array}{c}\text { Density } \\
\left(\mathrm{g} / \mathrm{cm}^{3}\right)\end{array}$ \\
\hline \multirow{2}{*}{ PAO 6 } & 40 & 31 & 0.81 \\
& 100 & 6 & 0.77 \\
\hline
\end{tabular}




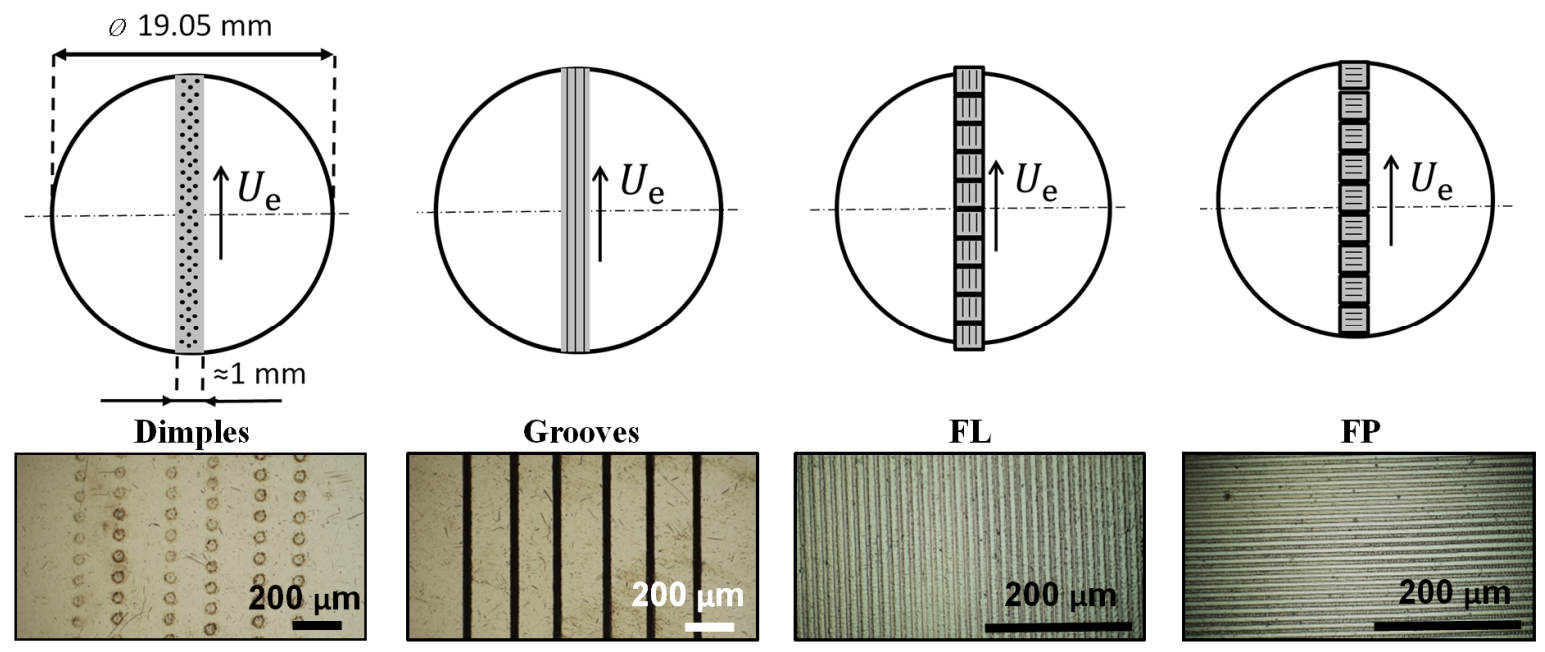

Fig. 1 Texturing configurations manufactured on smooth balls. $U_{\mathrm{e}}$ represents the entrainment speed.

(Quanta Ray Pro 290, Newport Spectra Physics) with a pulse duration of $10 \mathrm{~ns}$ and a Ti:Sapphire laser with a pulse duration of $100 \mathrm{fs}$. The textures were applied to the curved surface of steel balls. Here, the laser process offers the advantage of easily applying textures on curved surfaces. The only requirement is to integrate a rotation motor into the experimental setup which rotates the sample and therefore allows scanning the laser beam over the surface.

To create grooves (samples FL and FP in Fig. 1) with small periodicity, i.e., the distance from groove to groove, DLIP was employed. The primary nanosecond pulsed laser beam from Nd:YAG laser was split into two beams by a beam splitter. The laser had a wavelength of $355 \mathrm{~nm}$ and a repetition rate of $10 \mathrm{~Hz}$. The beam was overlapped by an optical setup on the ball surface where the two beams interfered with each other and thereby formed a sinusoidal line-like intensity distribution. The periodicity of the pattern is defined by the laser wavelength and the angle between the two interfering beams. The material was locally heated by local photo-thermal interaction between laser and metal. Material removal occurred at the intensity maxima positions of the interference pattern, creating the negative shape of the intensity distribution on the sample. The laser fluence was kept constant at $1.29 \mathrm{~J} / \mathrm{cm}^{2}$ for all experiments. Further details related to DLIP and the optical setup can be found in Refs. [36-38].

To create dimples as well as grooves with greater width and periodicity (samples Dimples and Grooves in Fig. 1) compared to the DLIP samples, a passively mode-locked ultrashort-pulsed Ti:Sapphire laser (Spitfire Pro XP, Newport Spectra Physics) was used. The laser had a repetition rate of $1 \mathrm{kHz}$ and a wavelength of $800 \mathrm{~nm}$. By using a lens (focal length of $100 \mathrm{~mm}$ ), the laser beam was focused on the surface of the ball. The desired texture pattern was created by moving the ball sample using a rotation stage. In the case of dimple texture, the distance between individual dimples was determined by the rotational velocity of the stage, whereas for the groove textures, the rotational speed had to be adapted to produce homogenous grooves [39]. In this context, in case of the groove pattern, individual laser spots with a diameter of $35 \mu \mathrm{m}$ were placed at a distance of $7 \mu \mathrm{m}$. The accumulated laser fluencies were 3.87 and $5.57 \mathrm{~J} / \mathrm{cm}^{2}$ for the dimples and the grooves, respectively.

The texturing speed in this laboratory environment depends on both the repetition rate of the laser and on the speed of the sample movement. For the samples FL and FP, the combination of the two parameters resulted in a texturing time of $12.0 \mathrm{~s}$ per sample. The texturing speed for the samples Dimples and Grooves was limited by the rotational speed of the rotation motor (maximum speed: $\left.10\left({ }^{\circ}\right) / \mathrm{s}\right)$, resulting in a texturing time of $218.9 \mathrm{~s}$ per samples with dimples and grooves. For higher rotational speed, the minimum texturing time of 7.2 and $89.7 \mathrm{~s}$ could be reached for the samples 
with dimples and grooves, respectively. However, it is worth mentioning that in industry, lasers with a much greater repetition rate and much higher speeds are realisable [40-42], which results in a considerable reduction of the texturing time.

\subsection{Tribological apparatus and test conditions}

Tribological tests were carried out using two different test rigs, being one for friction measurements (MTM2 rig) and another for film thickness evaluation (EHD rig). Both test rigs were purchased from PCS Instruments London.

The Mini Traction Machine (MTM2 rig) tribometer was used in the ball-on-disk configuration. A rotating ball is loaded against a rotating disk, in a temperaturecontrolled pot filled with lubricant, see Fig. 2(a). The ball and the disk are driven by two independent motors for continuous variation of speed and slideroll ratio. The $S R R$ is defined as

$$
S R R=100 \times \frac{U_{\text {Disk }}-U_{\text {Ball }}}{\left(U_{\text {Disk }}+U_{\text {Ball }}\right) / 2}=100 \times \frac{U_{\mathrm{s}}}{U_{\mathrm{e}}}
$$

where $U_{s}$ is the sliding speed and $U_{\mathrm{e}}$ is the entrainment (or rolling) speed. The $S R R$ ranges from pure sliding (SRR 200\%) to pure rolling ( $S R R$ $0 \%)$.
The tests were performed varying both the entrainment speed and sliding-rolling conditions to study a wide range of lubrication regimes and operational conditions. The test parameters are shown in Table 2. The maximum Hertzian contact pressure and the lubricant bath temperature were maintained constant during the tests at $0.6 \mathrm{GPa}$ and $40{ }^{\circ} \mathrm{C}$, respectively.

Film thickness was measured by optical interferometry, using an EHD rig. A steel ball is loaded against a coated glass disk, and white light is emitted on the contact between the bodies, see Fig. 2(b). The ball could be driven by the disk (pure rolling conditions) or by a shaft to permit continuous $S R R$ variations. Part of the light passing through the glass disk is reflected by a thin Cr layer at the bottom of the disk and another part is reflected by the steel ball after passing through the $\mathrm{SiO}_{2}$ spacer layer and the thin lubricant film. The two light beams are then recombined using a beam splitter, focused on a spectrometer, and captured by a white Charged Coupled Device (CCD) camera. It is possible to determine the central film thickness by measuring the light wavelength using the spectrometer [43-45]. Additionally, an RGB (red, green, and blue) colour camera could be utilised to
MTM2

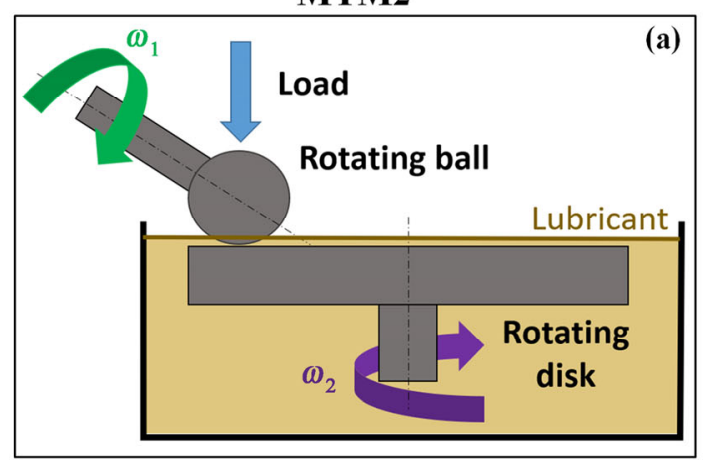

EHD

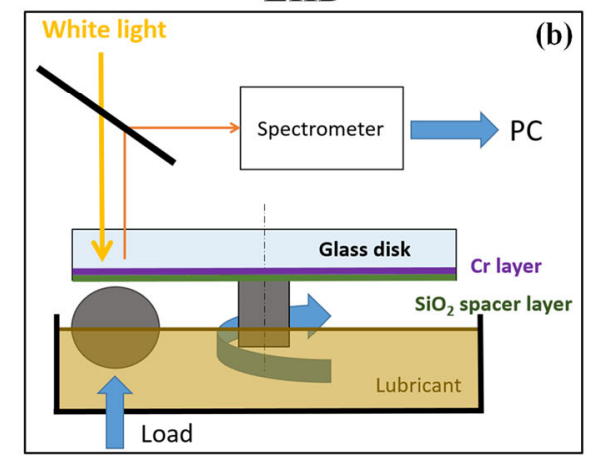

Fig. 2 Schematic representation of tribometers for (a) friction and (b) film thickness measurements.

Table 2 Test parameters adopted for friction (MTM2 rig) and film thickness (EHD rig) measurements. Note entrainment speed in descending order according to the sequence used in the experiments.

\begin{tabular}{cccccc}
\hline & Entrainment speed $U_{e}(\mathrm{~mm} / \mathrm{s})$ & $S R R(\%)$ & Temperature $\left({ }^{\circ} \mathrm{C}\right)$ & $\begin{array}{c}\text { Contact } \\
\text { pressure }(\mathrm{GPa})\end{array}$ & $\begin{array}{c}\text { Lubricant } \\
\text { MTM2 }\end{array}$ \\
EHD & $2,000-10$ & $5-20-50-80-100-120-150-180$ & 40 & 0.6 & PAO 6 \\
\hline
\end{tabular}


capture high-resolution images of the whole contact. This technique is named Spacer Layer Imaging Method (SLIM). A software is used to calibrate the system and produce a film thickness map of the whole contact. The EHD tribometer uses a white light source for the SLIM; however, brighter light is necessary for rough surfaces, as explained by Guégan et al. [46-48]. The authors proposed the use of two led sources (green and red) and the utilisation of a custom-made MatLab code to obtain the friction map over the whole contact area. Furthermore, this code determines the minimum and maximum film thickness as well as the amount of asperity contact (solid-to-solid contact) in case the system operates in mixed lubrication. Values of minimum film thickness are also used for evaluating the lift-off speed, defined as the minimum speed that promotes full surface separation, and consequently, the transition from mixed to full film lubrication regime [49]. Note that the high-speed camera takes the images at the same ball position using an encoder [46-48] for always analysing the same surface profile of the ball. This novel technique was applied to rough surfaces produced by lathe machining in Refs. [47, 48], and it is now applied for the first time in the available literature to textured samples in this contribution. The reader is referred to Refs. [46-48] for a detailed description of the technique.

The EHD tests were conducted using constant maximum Hertzian contact pressure (0.6 GPa) and temperature $\left(40{ }^{\circ} \mathrm{C}\right)$ under pure rolling conditions (SRR 0\%) varying the entrainment speed with logarithm increments to explore different lubrication regimes, see Table 2. Only pure rolling conditions were studied since the variation of sliding speed did not influence film thickness results significantly, as shown in Refs. [47, 48, 50].

\section{Results}

\subsection{Surface texture characterisation}

As mentioned in Section 2.2, the texture configurations named Dimples and Grooves were produced using the Ti:Sapphire laser, whereas the smaller grooves geometries FL and FP by Nd:YAG laser with the DLIP technique. The geometrical dimensions of surface textures were evaluated through a 3D optical profiler, and the main results are summarised in Table 3.

Note that the features produced by DLIP $(\mathrm{Nd}$ : YAG laser) presented a periodic pattern on almost half of the surface $(f \approx 50 \%)$, see Table 3 . On the

Table 3 Measured texture geometrical characteristics and laser parameters utilised for ball texturing.

\begin{tabular}{|c|c|c|c|c|}
\hline \multirow[b]{3}{*}{ Texture profile } & \multirow[t]{2}{*}{ Dimples } & \multicolumn{3}{|c|}{ Grooves } \\
\hline & & \multicolumn{2}{|c|}{ Longitudinal } & Perpendicular \\
\hline & & $U_{\mathrm{e}}$ & & $U_{\mathrm{e}}$ \\
\hline Texture name & Dimples & Grooves & FL & FP \\
\hline Features width $(\mu \mathrm{m})$ & $50 \pm 2$ & $35 \pm 2$ & $7 \pm 1$ & $7 \pm 1$ \\
\hline Features depth $(\mu \mathrm{m})$ & $0.1 \pm 0.02$ & $0.1 \pm 0.02$ & $0.4 \pm 0.02$ & $0.4 \pm 0.02$ \\
\hline Spacing, $p(\mu \mathrm{m})$ & $200 \pm 5$ & $200 \pm 5$ & $15 \pm 2$ & $15 \pm 2$ \\
\hline Area coverage, $f(\%)$ & $14 \pm 1$ & $21 \pm 1$ & $47 \pm 2$ & $47 \pm 2$ \\
\hline$R=$ depth/width & 0.002 & 0.003 & 0.06 & 0.06 \\
\hline Laser & Ti:Sapphire & Ti:Sapphire & Nd:YAG & Nd:YAG \\
\hline Pulse duration & $100 \mathrm{fs}$ & $100 \mathrm{fs}$ & $10 \mathrm{~ns}$ & $10 \mathrm{~ns}$ \\
\hline Laser fluence $\left(\mathrm{J} / \mathrm{cm}^{2}\right)$ & 3.87 & 5.57 & 1.29 & 1.29 \\
\hline
\end{tabular}


contrary, textures produced by Ti:Sapphire laser covered less area $(f<20 \%)$ and they were very shallow (depth $\approx 0.1 \mu \mathrm{m}$ ). Furthermore, features made with these fast laser techniques were not geometrically precise, especially for dimple configuration.

\subsection{Friction results}

The friction response measured using the MTM2 rig was plotted varying the entrainment speed $U_{\mathrm{e}}$ for fixed values of sliding-rolling, see Fig. 3. Four main $S R R$ conditions were selected, ranging from low (SRR 20\%) to high (SRR 180\%) sliding rates.

The texture configurations designed were not effective under full film condition, since all samples behaved similarly at high-speed conditions $\left(U_{\mathrm{e}}>\right.$ $600 \mathrm{~mm} / \mathrm{s}$ ), maintaining the coefficient of friction (COF) around 0.03 , see Fig. 3. The differences in the frictional behaviour became more relevant for lower entrainment speeds under mixed and boundary lubrication $\left(U_{\mathrm{e}}<600 \mathrm{~mm} / \mathrm{s}\right)$. In this region, the samples with longitudinal grooves increased friction compared to the smooth NP sample at different $S R R$ s, especially the configuration with a lower area coverage manufactured by Ti:Sapphire laser (Grooves), see Table 3 and Fig. 3. Perpendicular features (FP) improved tribological performance compared to longitudinal ones (Grooves and FL). However, they generally presented friction values close to reference smooth material (NP). Lastly, Dimples improved the tribological properties presenting the lowest frictional response in most configurations for low entrainment speeds.

In order to highlight the relative benefits (or lack thereof) in terms of performance obtained using different textured surfaces, the friction response of smooth surfaces (NP) was used as a reference to provide a direct comparison between the samples in terms of relative differences in COF. These values defined as Delta friction were obtained with the following expression:

$$
\text { Delta friction }(\%)=\left(\frac{\mathrm{COF}_{\text {Texture }}-\mathrm{COF}_{\mathrm{NP}}}{\mathrm{COF}_{\mathrm{NP}}}\right) \times 100
$$

where $\mathrm{COF}_{\text {Texture }}$ is the friction coefficient of textured surfaces (Dimples, Grooves, FP, and FL). Note that Delta friction assumes positive values for $\mathrm{COF}$ increment and negatives for COF reduction. COF results were plotted using percentage differences (Delta friction, \%) for each entrainment speed in Fig. 4. Every graph has different vertical axis ranges to

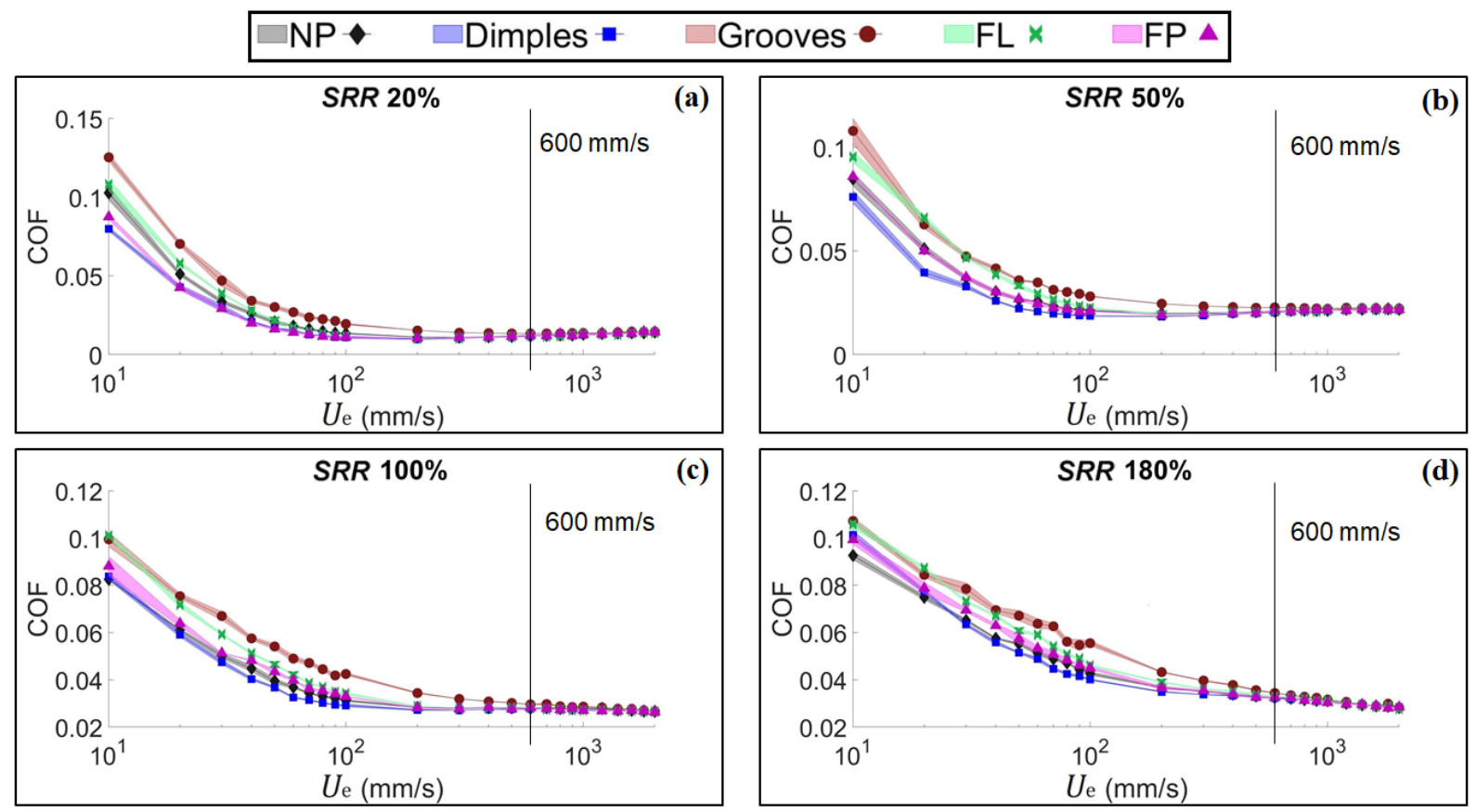

Fig. 3 COF vs. $U_{\mathrm{e}}$ using (a) $S R R 20 \%$, (b) $S R R 50 \%$, (c) $S R R 100 \%$, and (d) $S R R 180 \%$. The horizontal axis is in logarithmic scale. 
highlight the COF differences between texture configurations at fixed $S R R$ values rather than differences between $S R R$ s, see Fig. 4 .

As stated before, less dense longitudinal grooves (Grooves) presented worse results, increasing friction up to almost $60 \%$ for low entrainment speeds $\left(U_{\mathrm{e}}<\right.$ $600 \mathrm{~mm} / \mathrm{s}$ ). In comparison, more dense longitudinal grooves (FL), see Table 3, performed slightly better than Grooves, presenting an increase of approximately $20 \%$ compared to NP reference material. This behaviour is expected as already discussed in more detail in previous experimental and numerical studies [19, 51-53] carried out to compare the performance of different surface textures. In these cases, longitudinal pockets are shown to draw fluid from the contact rather than providing more fluid to increase the load support and reduce friction [19, 51-53]. Perpendicular grooves $(\mathrm{FP})$ slightly decreased friction $(\approx 10 \%)$ for low slide-roll ratio (SRR 20\%) and presented an increment in friction $(\approx 10 \%)$ for harsh sliding conditions (SRR 180\%). For mild sliding conditions (SRR 50\% and 100\%), FP and NP samples exhibited a similar response. Dimples reduced friction $(10 \%-$ $20 \%$ ) for all SRRs, especially for entrainment speeds below $100 \mathrm{~mm} / \mathrm{s}$. The reasons for these trends in the frictional performance are discussed in the next session, together with the film thickness results.

\subsection{Film thickness results}

To further investigate the tribological performance and the transition between lubrication regimes, the evolution of film thickness for textured and NP samples were evaluated for increasing entrainment speeds, ranging from 20 to $2,200 \mathrm{~mm} / \mathrm{s}$ using the described interferometry technique, see interferograms Fig. 5. Note that the lubricant flow direction is from left to right for each interferogram. Colour variations in the interferograms represent film thickness changes [43-45].

The classical EHL film thickness distribution (flat central area with the horse-shoe shape at high entrainment speeds [44]) could easily be detected for NP and textured samples with less dense texture configurations (Dimples and Grooves). On the contrary, the horse-shoe formation is only visible at high entrainment speeds $(2,200 \mathrm{~mm} / \mathrm{s})$ for surface textures manufactured by DLIP (FL and FP), probably due to rougher surfaces caused by dense texture configuration $(f \approx 47 \%$, see Table 3 ).

\section{- Dimples • Grooves $\times$ FL $\triangle \mathrm{FP}$}
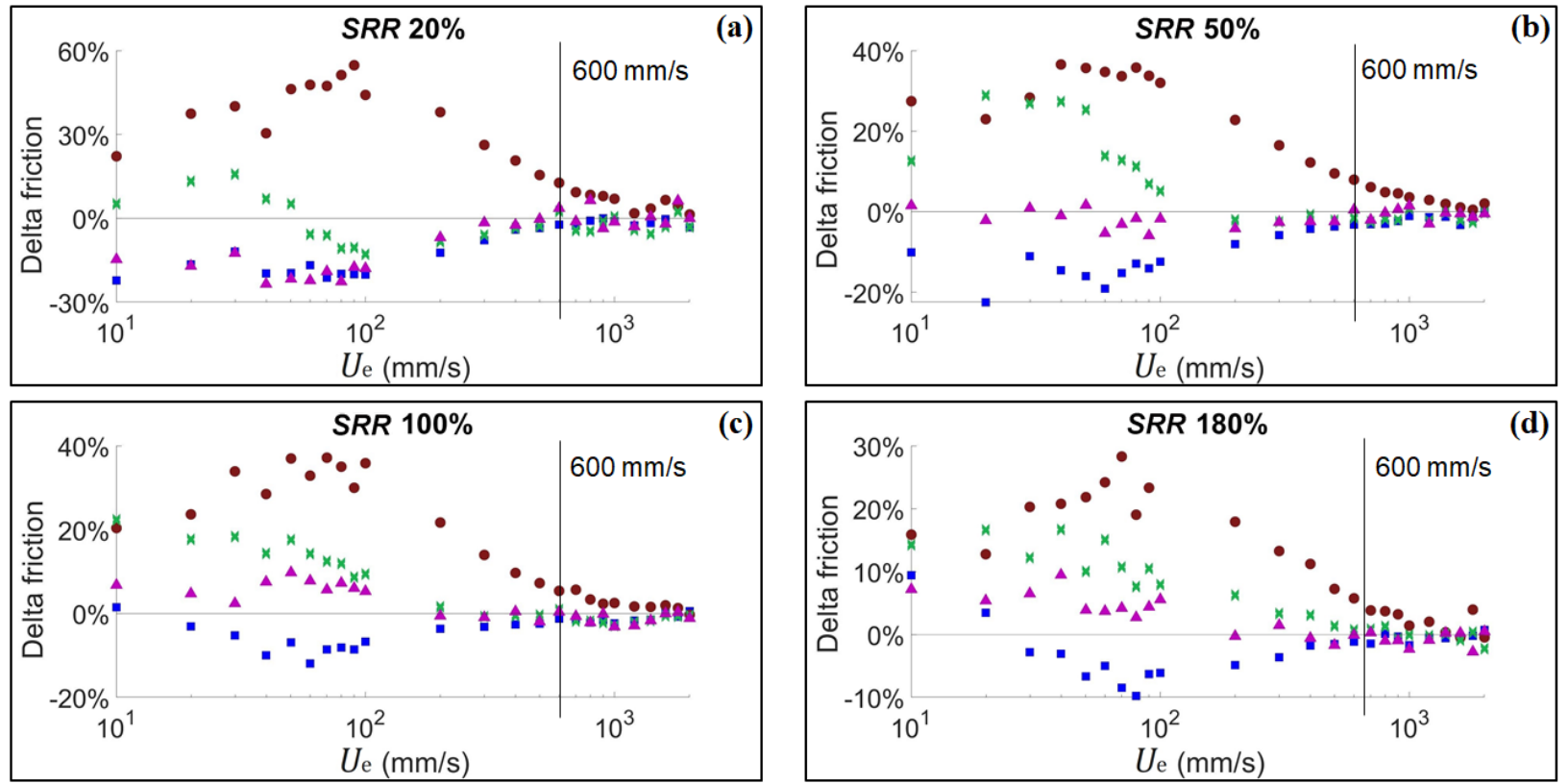

Fig. 4 Percentage differences in friction plotted as a function of $U_{\mathrm{e}}$ using (a) $S R R 20 \%$, (b) $S R R 50 \%$, (c) $S R R 100 \%$, and (d) SRR $180 \%$. The horizontal axis is in logarithmic scale. 


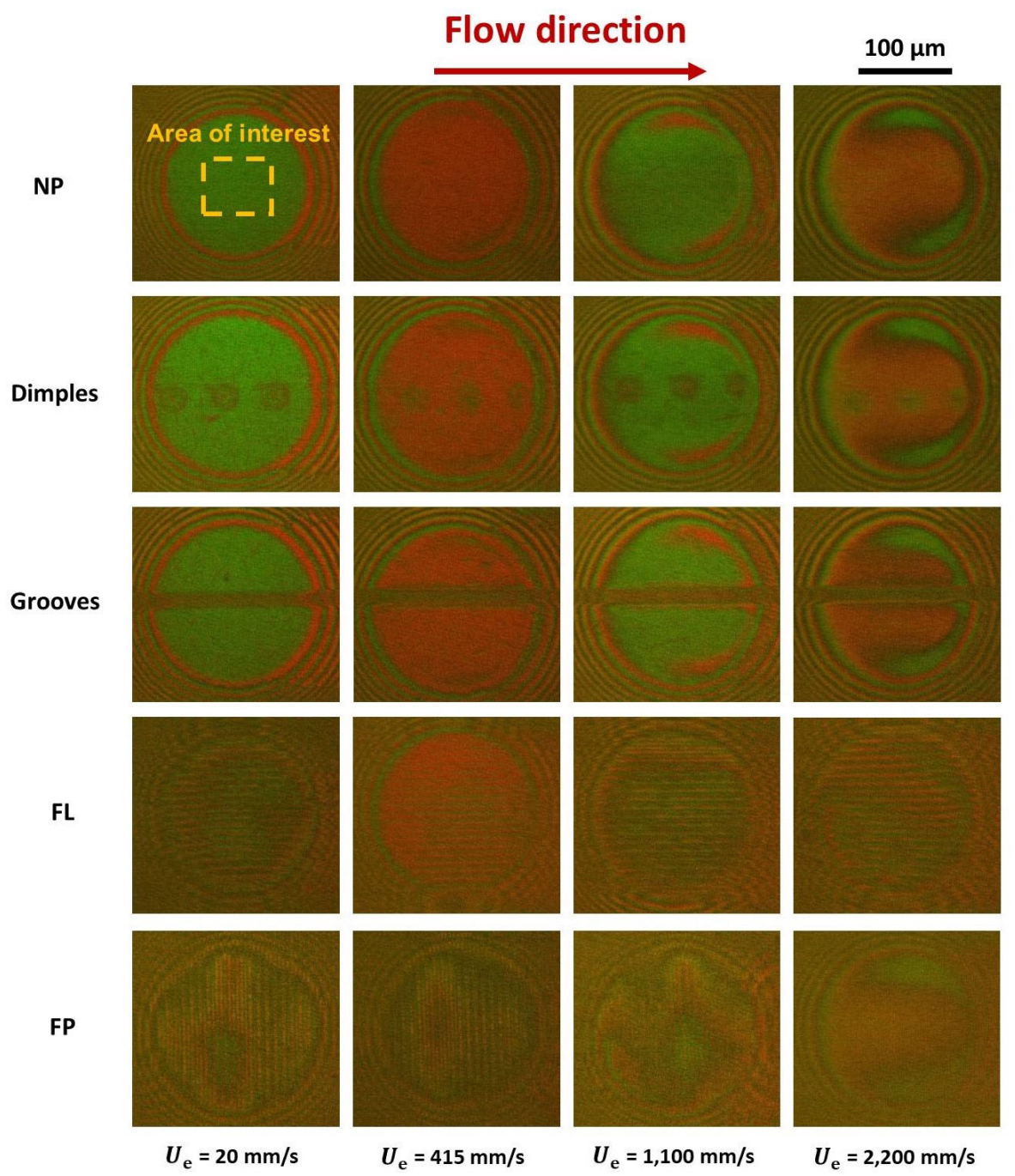

Fig. 5 Interferograms of NP and textured balls using different entrainment speeds under pure rolling conditions.

It should be noted from the results shown in Fig. 5 that, although the mounting of the ball was optimised, the direction of the Grooves, FL, and FP patterns is not perfectly longitudinal or perpendicular to the flow direction. This is due to small errors in the specimen assembling when the textures were manufactured; however, this is deemed to have a negligible effect on the results.

The minimum film thickness in the central region of each interferogram was measured by the custom-made MatLab routine designed for rough surfaces [46], see Fig. 5. In this case, minimum film thickness between surface asperities represents a key factor, since it is possible to identify the minimum speed for achieving full film conditions (lift-off speed). The variation of minimum film thickness at different entrainment speeds and liftoff values for textured and NP balls are depicted in Fig. 6.

Dimples promoted beneficial hydrodynamic conditions for lower speed (lift-off $\approx 311 \mathrm{~mm} / \mathrm{s}$ ), whereas perpendicular grooves (FP) presented similar behaviour of NP (lift-off $\approx 400 \mathrm{~mm} / \mathrm{s}$ ). Higher speeds $(\approx 600 \mathrm{~mm} / \mathrm{s})$ are necessary for full film lubrication conditions when using both longitudinal grooves (Grooves and FL) configurations. Note that minimum film thickness and friction values followed the same trend.

\section{Discussion}

According to the previous results, only Dimples 


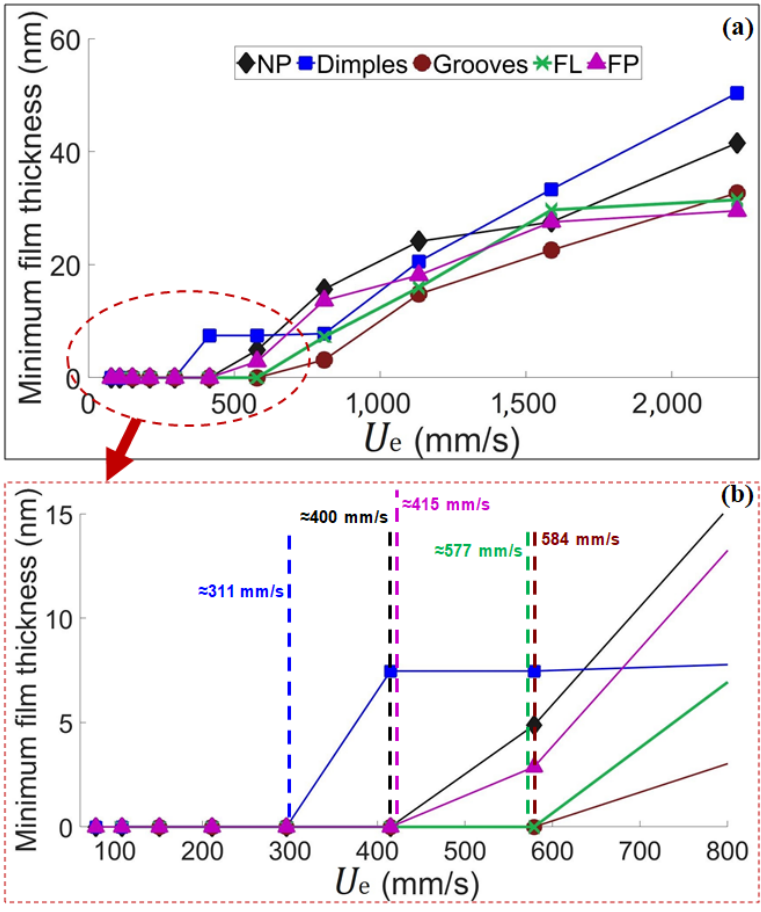

Fig. 6 (a) Measured minimum film thickness for NP and textured balls varying entrainment speed. (b) Details of the low-speed region $(<800 \mathrm{~mm} / \mathrm{s})$ and lift-off speed values (vertical dashed lines).

seemed to present positive micro-hydrodynamic bearing effects $[13,16,51]$, showing lower values of friction and promoting full film condition at lower speeds than smooth surfaces, see Figs. 4 and 6. Load-carrying capacity of the lubricating oil film is enhanced due to local changes in fluid flow and cavitation, as well as hydrodynamic pressure increments near the dimple and shear forces reduction over the textures. These effects are caused by elastic deformation and speed differences between bodies in EHL contact, as explained in Refs. [30, 32, 54]. This behaviour is particularly important for promoting wear reduction in real applications. In this context, the use of a textured surface with dimples could reduce asperity contacts between the rubbing surfaces compared to the untextured condition and, consequently, reducing wear and increasing component lifecycle $[19,52]$.

Perpendicular grooves (FP), even when designed using similar dimensional characteristics of Dimples (see Table 3), were not as effective since they generally showed similar results to the untextured surfaces (NP), see Figs. 4 and 6. The scarce micro-hydrodynamic benefits of FP could be justified by the much higher dimensions of the transversal grooves $(\approx 1 \mathrm{~mm})$ relative to the contact diameter $(\approx 170 \mu \mathrm{m})$. Therefore, more lubricant could flow transversally within the grooves, thus affecting the fluid pressure built-up close to the surface features, see Fig. 7(b). As mentioned above, this detrimental effect is generally evident for longitudinal configurations [9, 19], where features and entrainment speeds have the same direction and hence lubricant can more easily flow out of the contact zone through the grooves, see Fig. 7(c). For the Dimples configuration, besides the asymmetrical convergent-divergent geometry of the texture, the occurrence of the micro-hydrodynamic bearing mechanism is plausible since the dimple diameter is smaller than the contact area and, consequently, more lubricant can be retained in the interface, see Fig. 7(a).

The amount of asperity contact (solid-to-solid \%) was also measured using interferograms and analysed with a custom-made MatLab program [46], see Fig. 8. Longitudinal grooves (Grooves and FL) showed more contacting asperities in the mixed lubrication regime than perpendicular ones (FP) and Dimples. Not surprisingly, the proportion of solid-to-solid

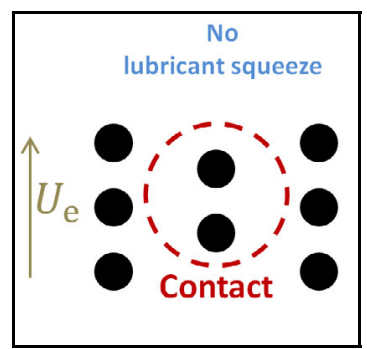

Dimples (a)

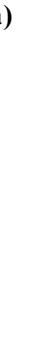

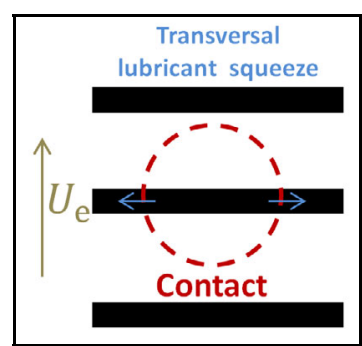

Perpendicular grooves

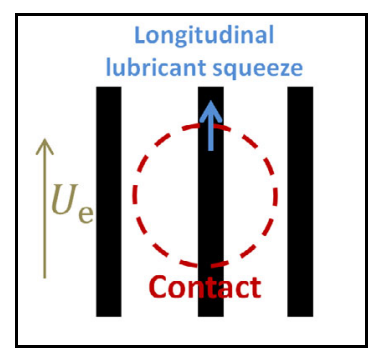

Longitudinal grooves (c)

(b)

Fig. 7 Schematic representation of lubricant behaviour for (a) dimples, (b) perpendicular, and (c) longitudinal groove texture configurations. 


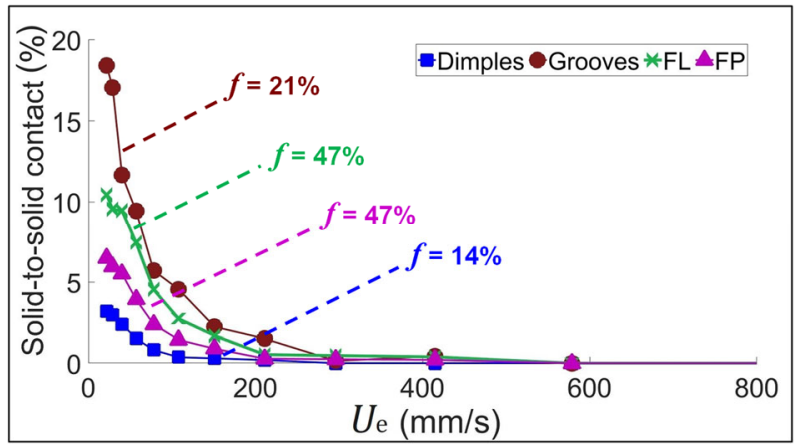

Fig. 8 Solid-to-solid contact (\%) of textured balls. $f$ indicates the texture area coverage parameter.

contact followed the same trend as the COF results and the measurements of the minimum film thickness, see Figs. 4, 6, and 8. In order to better understand the mechanisms involved, these results were evaluated also considering area coverage of textures $(f)$, see Table 3 .

Considering longitudinal grooves (Grooves and FL), the amount of asperity contact for Grooves was higher than for FL, due to a smaller area coverage for Grooves ( $f=21 \%$ compared to $47 \%$ ) and consequently a higher real contact area and more solid-to-solid contact. Both grooves manufactured by the Nd:YAG laser (FP and FL) covered almost half of the surface $(f=47 \%)$. However, perpendicular configuration FP offered less solid contact than FL, confirming more lubricant flow in the longitudinal direction compared to the transversal direction. This behaviour yielded a smaller load-carrying capacity and thus a smaller film thickness as well as a higher solid-to-solid contact, as shown in Figs. 7(b) and 7(c).

Dimples presented a lower solid-to-solid contact and consequently lower COF (see Figs. 4 and 6), even if the dimpled surface theoretically offered a higher real contact area (lowest values of $f$, see Table 3). These findings confirm the micro-hydrodynamic bearing effect of dimples in the mixed lubrication regime, by which the surfaces are separated further from each other, leading to smaller solid-to-solid contact and causing friction reduction while promoting full film conditions for lower speeds.

\section{Conclusions}

The tribological performance of curved surfaces, which were textured using fast laser techniques, has been evaluated. Friction and film thickness were measured by varying entrainment speed to scrutinise surface texture effects under different lubrication regimes. From the experimental results and the texture configurations studied, the following conclusions could be drawn:

The surface textures studied influenced the response of the system in mixed lubrication (below entrainment speed of $600 \mathrm{~mm} / \mathrm{s}$ ), whereas the full film lubrication regime was not affected.

Perpendicular features did not bring significant tribological benefit, probably due to their excessive transversal dimensions. The lubricant was able to flow within the grooves preventing a local hydrodynamic pressure increment and the consequent increase in load-carrying capacity.

Longitudinal grooves brought drawbacks to the performance of the curved surfaces compared to untextured, generally increasing solid-to-solid contact and friction. The lubricant could easily flow within the grooves since they are oriented in the same direction as the entrainment speed; therefore, local lubricant pressure built-up was obstructed.

It was shown that fast texturing techniques could be used effectively for improving the frictional performance of textured surfaces, as long as their design is linked to a clear understanding of the mechanisms responsible for such improvements. The accuracy of the textures' geometry does not constitute a crucial factor in this context.

\section{Acknowledgements}

This project was generously financed by the State of São Paulo Research Foundation, Brazil (FAPESP Grant Nos. 2016/25067-9 and 2017/21151-8) and the Brazilian National Council for Scientific and Technological Development (CNPq). G. BOIDI thanks the Austrian Research Promotion Agency (FFG) for the funding obtained by the Austrian COMETProgram (Project K2, InTribology, No. 872176) carried out at the "Excellence Centre of Tribology" (AC2T research $\mathrm{GmbH}$ ). The government of Lower Austria is gratefully acknowledged for financially supporting the endowed professorship tribology of Prof. C. 
GACHOT at the TU Wien (Grant No. WST3-F5031370/001-2017) in collaboration with AC2T research $\mathrm{GmbH}$. D. DINI also acknowledges the support received from the Engineering and Physical Sciences Research Council (EPSRC) via his Established Career Fellowship EP/N025954/1.

Open Access This article is licensed under a Creative Commons Attribution 4.0 International License, which permits use, sharing, adaptation, distribution and reproduction in any medium or format, as long as you give appropriate credit to the original author(s) and the source, provide a link to the Creative Commons licence, and indicate if changes were made.

The images or other third party material in this article are included in the article's Creative Commons licence, unless indicated otherwise in a credit line to the material. If material is not included in the article's Creative Commons licence and your intended use is not permitted by statutory regulation or exceeds the permitted use, you will need to obtain permission directly from the copyright holder.

To view a copy of this licence, visit http://creativecommons.org/licenses/by/4.0/.

\section{References}

[1] Hamilton D B, Walowit J A, Allen C M. A theory of lubrication by microirregularities. J Basic Eng 88(1): 177-185 (1966)

[2] Martz L S. Preliminary report of developments in interrupted surface finishes. Proc Inst Mech Eng 161(1): 1-9 (1949)

[3] von Turkovich B F, Roubik J R, Gilbert W W, Ham I, Oxford C J Jr, Pickrell A L, Wilson G F, Boulger F W, Hahn R S, Krabacker E J, et al. 1963 review of metal processing literature. J Eng Ind 87(1): 85-96 (1965)

[4] Putignano C, Scarati D, Gaudiuso C, Di Mundo R, Ancona A, Carbone G. Soft matter laser micro-texturing for friction reduction: An experimental investigation. Tribol Int 136: 82-86 (2019)

[5] Fowell M, Olver A V, Gosman A D, Spikes H A, Pegg I. Entrainment and inlet suction: Two mechanisms of hydrodynamic lubrication in textured bearings. $J$ Tribol 129(2): 336-347 (2007)

[6] Scaraggi M, Mezzapesa F P, Carbone G, Ancona A, Tricarico L. Friction properties of lubricated lasermicrotextured-surfaces: An experimental study from boundary- to hydrodynamic-lubrication. Tribol Lett 49(1): 117-125 (2013)

[7] Gropper D, Wang L, Harvey $T$ J. Hydrodynamic lubrication of textured surfaces: A review of modeling techniques and key findings. Tribol Int 94: 509-529 (2016)

[8] Boidi G, Rodrigues da Silva M, Profito F J J, Machado I F. Using machine learning Radial Basis Function (RBF) method for predicting lubricated friction on textured and porous surfaces. Surf Topogr Metrol Prop, in press, DOI 10.1088/2051-672X/abae13.

[9] Gachot C, Rosenkranz A, Hsu S M, Costa H L. A critical assessment of surface texturing for friction and wear improvement. Wear 372-373: 21-41 (2017)

[10] Pettersson U, Jacobson S. Influence of surface texture on boundary lubricated sliding contacts. Tribol Int 36(11): 857-864 (2003)

[11] Kovalchenko A, Ajayi O, Erdemir A, Fenske G, Etsion I. The effect of laser texturing of steel surfaces and speed-load parameters on the transition of lubrication regime from boundary to hydrodynamic. Tribol Trans 47(2): 299-307 (2004)

[12] Rosenkranz A, Heib T, Gachot C, Mücklich F. Oil film lifetime and wear particle analysis of laser-patterned stainless steel surfaces. Wear 334-335: 1-12 (2015)

[13] Yu H W, Wang X L, Zhou F. Geometric shape effects of surface texture on the generation of hydrodynamic pressure between conformal contacting surfaces. Tribol Lett 37(2): 123-130 (2010)

[14] Kovalchenko A, Ajayi O, Erdemir A, Fenske G, Etsion I. The effect of laser surface texturing on transitions in lubrication regimes during unidirectional sliding contact. Tribol Int 38(3): 219-225 (2005)

[15] Olver A V, Fowell M T, Spikes H A, Pegg I G. 'Inlet suction', a load support mechanism in non-convergent, pocketed, hydrodynamic bearings. Proc Inst Mech Eng Part J: J Eng Tribol 220(2): 105-108 (2006)

[16] Wang X L, Zhang H, Hsu S. The effects of dimple size and depth on friction reduction under boundary lubrication pressure. In ASME/STLE 2007 International Joint Tribology Conference, San Diego, USA, 2007: 24-26.

[17] Profito F J, Vlădescu S C, Reddyhoff T, Dini D. Transient experimental and modelling studies of laser-textured micro-grooved surfaces with a focus on piston-ring cylinder liner contacts. Tribol Int 113: 125-136 (2017)

[18] Qui Y, Khonsari M M. On the prediction of cavitation in dimples using a mass-conservative algorithm. J Tribol 131(4): 041702 (2009)

[19] Vlădescu S C, Olver A V, Pegg I G, Reddyhoff T. The effects of surface texture in reciprocating contacts-An experimental study. Tribol Int 82: 28-42 (2015) 
[20] Ibatan T, Uddin M S, Chowdhury M A K. Recent development on surface texturing in enhancing tribological performance of bearing sliders. Surf Coatings Technol 272: 102-120 (2015)

[21] Costa H L, Hutchings I M. Some innovative surface texturing techniques for tribological purposes. Proc Inst Mech Eng Part J: J Eng Tribol 229(4): 429-448 (2015)

[22] Lasagni A F, Gachot C, Trinh K E, Hans M, Rosenkranz A, Roch T, Eckhardt S, Kunze T, Bieda M, Günther D, Lang V, Mücklich F. Direct laser interference patterning, 20 years of development: From the basics to industrial applications. In Proceedings of SPIE 10092, LaserBased Micro- and Nanoprocessing XI, San Francisco, USA, 2017: 1009211.

[23] Grützmacher P G, Rosenkranz A, Szurdak A, Gachot C, Hirt G, Mücklich F. Effects of multi-scale patterning on the run-in behavior of steel-alumina pairings under lubricated conditions. Adv Eng Mater 20(1): 1700521 (2018)

[24] Grützmacher P G, Profito F J, Rosenkranz A. Multiscale surface texturing in tribology-Current knowledge and future perspectives. Lubricants 7(11): 95 (2019)

[25] Sudeep U, Tandon N, Pandey R K. Performance of lubricated rolling/sliding concentrated contacts with surface textures: A review. J Tribol 137(3): 031501 (2015)

[26] Rosenkranz A, Grützmacher P G, Gachot C, Costa H L. Surface texturing in machine elements-A critical discussion for rolling and sliding contacts. Adv Eng Mater 21(8): 1900194 (2019)

[27] Choo J W, Olver A V, Spikes H A. The influence of transverse roughness in thin film, mixed elastohydrodynamic lubrication. Tribol Int 40(2): 220-232 (2007)

[28] Venner C H, Lubrecht A A. Numerical simulation of a transverse ridge in a circular EHL contact under rolling/sliding. J Tribol 116(4): 751-761 (1994)

[29] Ehret P, Dowson D, Taylor C M. Waviness orientation in EHL point contact. Tribol Ser 31: 235-244 (1996)

[30] Marian M, Grützmacher P, Rosenkranz A, Tremmel S, Mücklich F, Wartzack S. Designing surface textures for EHL point-contacts-Transient 3D simulations, metamodeling and experimental validation. Tribol Int 137: 152-163 (2019)

[31] Gao L M, De Boer G, Hewson R. The role of micro-cavitation on EHL: A study using a multiscale mass conserving approach. Tribol Int 90: 324-331 (2015)

[32] Mourier L, Mazuyer D, Ninove F P, Lubrecht A A. Lubrication mechanisms with laser-surface-textured surfaces in elastohydrodynamic regime. Proc Inst Mech Eng Part J: J Eng Tribol 224(8): 697-711 (2010)

[33] Rosenkranz A, Szurdak A, Gachot C, Hirt G, Mücklich F. Friction reduction under mixed and full film EHL induced by hot micro-coined surface patterns. Tribol Int 95: 290-297 (2016)

[34] Krupka I, Hartl M, Zimmerman M, Houska P, Jang S. Effect of surface texturing on elastohydrodynamically lubricated contact under transient speed conditions. Tribol Int 44(10): 1144-1150 (2011)

[35] Boidi G, Tertuliano I S, Profito F J, de Rossi W, Machado I F. Effect of laser surface texturing on friction behaviour in elastohydrodynamically lubricated point contacts under different sliding-rolling conditions. Tribol Int 149: 105613 (2019)

[36] Mücklich F, Lasagni A, Daniel C. Laser interference metallurgy-Using interference as a tool for micro/nano structuring. Int J Mater Res 97(10): 1337-1344 (2006)

[37] Grützmacher P G, Rosenkranz A, Szurdak A, König F, Jacobs G, Hirt G, Mücklich F. From lab to applicationImproved frictional performance of journal bearings induced by single- and multi-scale surface patterns. Tribol Int 127: 500-508 (2018)

[38] Lasagni A, Holzapfel C, Weirich T, Mücklich F. Laser interference metallurgy: A new method for periodic surface microstructure design on multilayered metallic thin films. Appl Surf Sci 253(19): 8070-8074 (2007)

[39] Grützmacher P G, Rosenkranz A, Gachot C. How to guide lubricants-Tailored laser surface patterns on stainless steel. Appl Surf Sci 370: 59-66 (2016)

[40] Cheng H H, Wang W L, Zhou Y, Qiao T, Lin W, Xu S H, Yang $\mathrm{Z}$ M. $5 \mathrm{GHz}$ fundamental repetition rate, wavelength tunable, all-fiber passively mode-locked Yb-fiber laser. Opt Express 25(22): 27646-27651 (2017)

[41] Schille J, Schneider L, Streek A, Kloetzer S, Loeschner U. High-throughput machining using high average power ultrashort pulse lasers and ultrafast polygon scanner. In Proceedings of SPIE 9736, Laser-Based Micro- and Nanoprocessing $X$, San Francisco, USA, 2016: 97360R.

[42] Lasagni A, Benke D, Kunze T, Bieda M, Eckhardt S, Roch T, Langheinrich D, Berger J. Bringing the direct laser interference patterning method to industry: A one tool-complete solution for surface functionalization. $J$ Laser Micro/Nanoeng 10(3): 340-344 (2015)

[43] Cann P M, Spikes H A, Hutchinson J. The development of a spacer layer imaging method (SLIM) for mapping elastohydrodynamic contacts. Tribol Trans 39(4): 915-921 (1996)

[44] Johnston G J, Wayte R, Spikes H A. The measurement and study of very thin lubricant films in concentrated contacts. Tribol Trans 34(2): 187-194 (1991)

[45] Luo J B, Wen S Z, Huang P. Thin film lubrication. Part I. Study on the transition between EHL and thin film lubrication using a relative optical interference intensity technique. Wear 194(1-2): 107-115 (1996) 
[46] Guégan J. Experimental investigation into the influence of roughness on friction and film thickness in EHD contacts. Ph.D. Thesis. London (UK): Imperial College London, 2015.

[47] Guégan J, Kadiric A, Gabelli A, Spikes H. The relationship between friction and film thickness in EHD point contacts in the presence of longitudinal roughness. Tribol Lett 64(3): 33 (2016)

[48] Guégan J, Kadiric A, Spikes H. A study of the lubrication of EHL point contact in the presence of longitudinal roughness. Tribol Lett 59(1): 22 (2015)

[49] Heemskerk $R$ S, Vermeiren $K$ N, Dolfsma $H$. Measurement of lubrication condition in rolling element bearings. ASLE Trans 25(4): 519-527 (1982)

[50] Rycerz P, Kadiric A. The influence of slide-roll ratio on the extent of micropitting damage in rolling-sliding

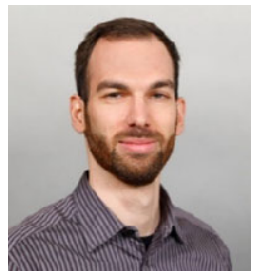

G. BOIDI. He is a scientist at AC2T Research GmbH (Austria) from 2019. He received his Ph.D. degree from the University of São Paulo (USP - Brazil) in 2019, where he studied the tribological effect of surface irregularities (laser texturing and porosity

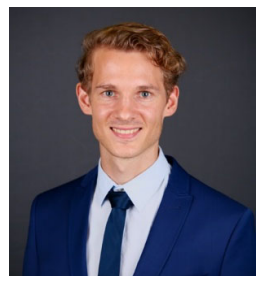

P.G. GRÜTZMACHER. He received his Ph.D. in materials science in 2019 from Saarland University under the guidance of Prof. Dr. Frank Mücklich (Institute of Functional Materials) for the tribological investigation of multi-scale surface textures. Since

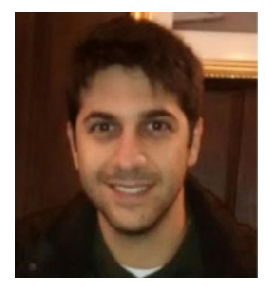

F.J. PROFITO. He is an assistant professor in mechanical engineering at Polytechnic School of the University of São Paulo (Brazil) and a member of the Surface Phenomena Laboratory in the same institution. His researches are focused on general topics of tribology, especially on lubrication and contact mechanics, computational tribology, surface topography, journal, and sliding bearings, internal combustion engines, and automotive transmissions. He received his B.Eng. (2008), M.Sc. contacts pertinent to gear applications. Tribol Lett 67(2): 63 (2019)

[51] Rosenkranz A, Costa H L, Profito F, Gachot C, Medina $\mathrm{S}$, Dini D. Influence of surface texturing on hydrodynamic friction in plane converging bearings-An experimental and numerical approach. Tribol Int 134: 190-204 (2019)

[52] Vlădescu S C, Ciniero A, Tufail K, Gangopadhyay A, Reddyhoff $\mathrm{T}$. Looking into a laser textured piston ring-liner contact. Tribol Int 115: 140-153 (2017)

[53] Scaraggi M, Carbone G, Dini D. Experimental evidence of micro-EHL lubrication in rough soft contacts. Tribol Lett 43(2): 169-174 (2011)

[54] Křupka I, Hartl M. The effect of surface texturing on thin EHD lubrication films. Tribol Int 40(7): 1100-1110 (2007)

in sintered materials) under the guidance of Prof. Izabel Fernanda Machado. He was a visitor Ph.D. student for one year at Imperial College London (2018-2019) under the guidance of Prof. Daniele Dini. His research interests involved surface texturing, tribology of powder metallurgy, spark plasma sintering, and sintered bearings and gears.

2019, he has been working in Prof. Dr. Carsten Gachot's tribology group at TU Vienna as a postdoctoral researcher. His current research interests focus on nearsurface microstructural development of polycrystalline metals during sliding, tribological mechanisms of 2D materials, and surface engineering.

(2010), and D.Sc. (2015) in mechanical engineering from the Polytechnic School of the University of São Paulo. His M.Sc. and D.Sc. researches were focused on the development of mathematical models and numerical simulation tools for predicting the mixed-elastohydrodynamic lubrication performance of journal and sliding bearing systems and piston ring-cylinder liner assemblies. He has three years of experience in the automotive industry as an M.Sc. researcher at MAHLE Research Centre in Brazil. He also spent one year at Imperial College London as a visiting Ph.D. researcher. $\mathrm{He}$ also 
earned two thesis awards from the University of São Paulo (USP/Brazil) and the Coordination for

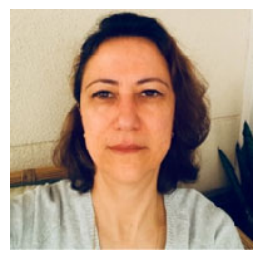

I.F. MACHADO. She got her Ph.D. degree (1999) from the University of São Paulo (USP). In 2001, she became a faculty member of USP, and currently holds the position of a associate professor at USP.

She researched materials characterization, mechanical and tribological behavior of materials focusing on

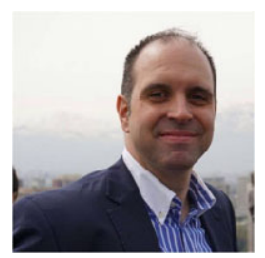

C. GACHOT. He received his Ph.D. degree from the Saarland University in Germany in 2012 where he studied the effects of laser interference patterning on the microstructure and topography of metallic surfaces with a focus on tribological applications under Prof. Dr. Frank Mücklich and Prof. Dr. Martin H. Müser. For this work, he was awarded the European Honda initiation grant in

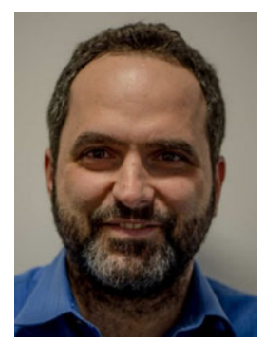

D. DINI. He is the head of the Tribology Group at Imperial College London. Prior to joining Imperial College in 2006, he completed his D.Phil. degree in the Department of Engineering Science at the University of Oxford (2004). His individual research portfolio supports a large team of researchers focused on studies related to the Improvement of Higher Level Education Personnel (Capes/Brazil).

applications and manufacturing process, which were conducted both experimentally and numerically simulated. She has also researched sintering by means Spark Plasma Sintering (SPS), using metal alloys and ceramics for different applications. She was a visiting professor at Trento University (Italy) in 2008 and the University of California-Davis in 2020.

2011. He was an academic visitor at the tribology Group at the Imperial College London and is currently the head of the tribology research Group at the Vienna University of Technology. Additionally, he is a visiting professor at the Pontifical Catholic University in Santiago de Chile and the chief editor of the peer-reviewed journal Industrial Lubrication and Tribology of the Emerald Publishing Group Leeds UK.

the modelling of tribological systems and materials. Most of these projects are multidisciplinary and range from atomic and molecular simulation of lubricants, additives and surfaces to the modelling of systems, such as machine and biomedical components. His group performs fundamental research, while successfully supporting the application of tribology in industry. 